\title{
El posicionamiento de una marca deportiva (Brand positioning)
}

\section{The positioning of a sports brand}

Daniel Ortiz Gonzales. ${ }^{1}$, María Francisca Torres Cedeño. ${ }^{2}$, \& Holger Arturo Intriago Mairongo. ${ }^{3}$

\section{DOI: https://doi.org/10.33262/visionariodigital.v3i3.611}

\begin{abstract}
.
Sport is a market that was able to generate more than 80 billion US Dollars last year. Because of that reason, sport brands strategies need to be increasingly studied in detail. One of the strategies that offers more positive results inside the sport brands is the brand positioning and its effects into the consumer. In this work you can find the common phases and the main characteristics to achieve an excellent positioning and to be successful getting relevance inside the consumer's mind. In addition, there are also analyses of the general positioning that have two powerful companies like Nike and Puma. The information explained is complemented and is compared to a questionnaire done by 160 people about the sport brands positioning.
\end{abstract}

Keywords: Reverse logistics (L81), fast fashion (L81), competitive advantage (L25)

\section{Resumen}

El deporte es un sector que el año pasado fue capaz de generar más de 80 billones de dólares americanos. Es por ello que las estrategias que lleven a cabo las marcas

${ }^{1}$ Universidad de Barcelona, Facultad de Ciencias Administrativas y Económica, Barcelona - España, ortizgd@ub.edu

2 Universidad Técnica Luis Vargas Torres, Sede Santo Domingo, Facultad de Ciencias Administrativas y Económicas, Santo Domingo - Ecuador, ikatorrescedeño@gmail.com

3 Universidad Técnica Luis Vargas Torres, Sede Santo Domingo, Facultad de Ciencias Administrativas y Economicas, Santo Domingo - Ecuador, holger.intriago@utelvt.edu 
deportivas deben ser cada vez más estudiadas al detalle. Una de las estrategias que mayores resultados positivos ofrece a las marcas deportivas es el posicionamiento que estas logran en el consumidor. En este trabajo se analizan las fases y características que se deben dar para lograr un posicionamiento óptimo y conseguir así que una marca tenga relevancia en la mente de una persona. También se lleva a cabo un análisis de los posicionamientos que tienen dos empresas tan potentes como Nike y Puma. Toda la información que se explica en el trabajo quedará complementada y comparada con los resultados de un cuestionario hecho a 160 personas sobre el posicionamiento de las marcas deportivas.

Palabras Claves: logística inversa (L81), moda rápida (L81), ventaja competitiva (L25)

\section{Introducción}

\section{El Marketing Deportivo}

En el pasado año se estimó que la industria global del deporte ingresó alrededor de 80 billones de dólares teniendo en cuenta el ticketing, derechos audiovisuales y patrocinios; de haber incluido también todos los productos deportivos que se crean alrededor del deporte (equipamiento deportivo, gastos en fitness y salud...) la cifra llegaría hasta los 700 billones de dólares, el 1\% del PIB mundial (Kearney, A., 2015). Por lo tanto, es imposible no entender la importancia que tiene el deporte como 'money-raiser' en la economía mundial. El deporte está cada vez más comercializado y profesionalizado con el paso de los años. Las empresas y negocios creados alrededor de la industria deportiva tienen que competir contra otras industrias de ocio o incluso competir dentro del mismo sector entre sí para alcanzar su posición deseada en el mercado. Un mundo comercial y económico cada vez más agresivo ha hecho que el marketing se haya convertido en una herramienta indispensable dentro de las estrategias del mundo del deporte hoy en día. Pero, ¿cuándo aparece el marketing deportivo? Está claro que actualmente el deporte ocupa un papel fundamental en las vías de entretenimiento existentes, y son las marcas quienes se aprovechan de ello y nos invaden con millones de impactos en nuestro día a día. Pero, ¿cuándo empezaron las marcas a darse cuenta del potencial que tenían utilizando el deporte como medio de promoción? ¿O que las comercializaciones de sus productos deportivos iban a conseguir mayores ingresos? Puede parecer extraño, pero podemos remontarnos hasta la Antigua Roma para empezar a observar pequeñas reseñas del marketing deportivo como lo conocemos hoy en día. Y es que los patriarcas romanos esponsorizaban a los gladiadores más fuertes y populares por la misma razón que se hace en el mundo actual: para ganar el aprecio del público En términos actuales podríamos decir que estaban buscando su posicionamiento en el mercado, ser reconocidos por los "clientes" y ganar popularidad para ser aprovechado en futuras ocasiones. Pero es en 
la segunda mitad del siglo XX cuando el marketing deportivo moderno se expande. En la década de los '70, surge la explotación de la publicidad de la industria tabaquera que utiliza el deporte como medio de promoción. Carreras de coches, torneos de tenis, etc. pusieron el nombre de marcas de cigarrillos enfrente de los espectadores. En los Juegos Olímpicos de 1984 de Los Ángeles (EEUU), de la mano de Juan Antonio Samaranch, nace el marketing deportivo olímpico. Las marcas revalorizan el deporte y empiezan a moverse grandes cantidades de dinero alrededor del mismo. El COI (Comité Olímpico Internacional) creó un sistema de patrocinios permanentes (The Olympic Partners) para que las marcas tuvieran la oportunidad de comercializar sus productos con motivo de las Olimpiadas. Estos ingresos pasaron de 7 millones de dólares en 1976 a 96 millones de dólares entre 1985 y 1988; alcanzando los 579 millones en el periodo 1997-2000, y que necesidades. Sin embargo, antes de cualquier venta, ese producto o servicio deportivo necesita tener un lugar en la mente del consumidor para así conseguir atraerlo cuando este tenga un estímulo del producto. Este proceso se denomina 'branding' y cuando la marca deportiva ha obtenido un lugar firme en la mente del consumidor entonces se dice que se trata de una marca posicionada (Smith, A., 2008). El marketing deportivo tiene una gran diferencia que lo dista completamente del marketing general que hay en otras industrias, y es que el deporte mueve emociones y pasiones en la gente (Mullin, 1983). El deporte puede estimular una respuesta emocional en el consumidor que es incapaz de darse con otros productos. Por ello, la conexión existente entre la psicología y el marketing deportivo es mucho mayor en este caso y se tratará de promover el interés, participación y consumo de los productos y servicios deportivos que pueden conectarse con el comportamiento de un fan (Ratten V., 2015).

Estos procesos son muy importantes en un mercado como el deportivo. Si una marca es atractiva para los consumidores, estos comprarán sus productos con el de los competidores y con el tiempo harán de ellos activos muy valiosos. En el deporte, muchas empresas compiten en los mismos mercados con productos muy semejantes, por lo tanto, la imagen preestablecida en la mente de los consumidores es de suma importancia. Sin embargo, pocas marcas son tan poderosas como para protegerla de la competencia que hacen otras marcas introduciendo productos similares a precios sustancialmente inferiores o con mejores prestaciones. Es por ello que las estrategias de marketing dentro de las empresas son decisivas, ya que normalmente asegura que las marcas de la compañía no se vean perjudicadas por alguno de esos factores (Murphy J., 1988). Esta técnica de posicionar el producto en la mente del consumidor comienza a nombrarse y estudiar en los años 70 de la mano J. Trout (1969), en cuyo estudio habla del posicionamiento firme que tenía el líder del mercado informático IBM, y como sus competidores no tenían ninguna posibilidad de superarle a pesar de igualar sus productos. Anteriormente a esta fecha, todo se basaba en crear una simple imagen de empresa basada en la publicidad del producto sin tener en cuenta a fondo el target o el tipo de mercado. El posicionamiento, un elemento que se desarrolla en la mente, coincide con el desarrollo que tuvo la psicología a partir de 1960 en múltiples 
campos, entre ellos el de la publicidad, donde se empieza a persuadir al consumidor satisfaciendo sus deseos y placeres (Rom, J. y Sabaté, J., 2007). Sin embargo, otros autores como Porter (1996) o Mintzberg (1999) hablaban de la posibilidad de hablar de un posicionamiento más 'físico' que 'mental' atribuyéndolo en el mercado en vez de en la mente de los consumidores. Este se referiría al lugar que ocupa una empresa en un mercado determinado, y que aquellas que ocupen una mejor posición, obtendrán mayores beneficios económicos y podrán seguir aumentando esa posición de liderazgo.

Estaban basadas básicamente en indicadores económicos. Son datos objetivos, pero difíciles de correlacionar con la imagen real que posee la marca. Vivimos en un mundo globalizado, con millones de impactos diarios, empresas deportivas con Core Business semejantes, productos y servicios muy similares... Es por ello que a la hora de posicionarse no debemos preguntarnos en qué somos mejores, sino en qué somos diferentes.

\section{- Marco Teórico Referencial}

Para que una idea logre entrar en la mente del consumidor se deben llevar a cabo estrategias muy bien preparadas dado que solo funcionan en el momento justo y bajo las circunstancias concretas. Una empresa puede tener un gran producto, un alto volumen de ventas, buena promoción y finalmente no lograr su objetivo o fracasar en el intento (Ries y Trout, 2008). La empresa debe tener muy claro lo que quiere transmitir al consumidor, y antes de adentrarnos a ver los pasos que se deben de seguir para lograr el posicionamiento deseado. Según Ries y Trout (2008) se debían seguir los siguientes pasos:

1. Encontrar una idea sencilla, fácil de transmitir y diferente de la competencia.

2. Respaldar la idea con hechos reales para que los consumidores puedan comprobar su veracidad.

3. Diseñar un plan de implantación (comunicación) que informe de esa diferencia al target cliente. Según Geoffrey Moore (Crossing the Chasm, 2014) hay que tener en cuenta cuatro elementos esenciales a la hora de establecer el posicionamiento de una marca:

A. Target cliente: Se debe entender a la perfección a quien va dirigido el producto: edad, sexo, actitudes, pasiones... De esta forma la marca conseguirá entender mejor a quién va dirigido su producto y adecuar la manera en cómo se transmite la idea de posicionamiento.

B. Definición del mercado: Saber a la perfección en qué tipo de mercado está entrando la marca. Contra quién compite, quién es el líder y dónde quiere estar esta marca dentro del mercado. En qué categoría quiere tener relevancia para los clientes. Moore define el mercado como "un conjunto de potenciales clientes para un determinado conjunto de productos o servicios que corresponden a necesidades o intereses 
comunes a todos ellos y que emplean como referencia a otros miembros del mercado en el momento de efectuar una decisión de compra".

C. Promesa de la marca (Brand promise): Son los beneficios emocionales y racionales que desearía el target cliente si consume esa marca; y que los competidores no son capaces de ofrecer. Esos beneficios deberán ser fiel reflejo de la imagen que la marca tiene en la mente del consumidor y mantenerse una vez el cliente adquiere sus productos.

D. "Reason to believe": Una marca debe apelarse siempre a su identidad. Enfocarse en lo que promete la marca. Las 'razones para creer' son las pruebas convincentes y tangibles que el cliente percibe sobre la promesa de la marca. Ej. Creación de un eslogan que es reflejo del posicionamiento global de la marca o la utilización de deportistas como Nike hizo con Michael Jordán.

- Número de páginas del artículo entre 10 y 22 paginas

\section{Metodología.}

El tamaño de la muestra de este estudio se situó 160 encuestados de segunda etapa, es decir, personas de 15 años en adelante. Básicamente no se han cogido sujetos menores a esa edad dado que el posicionamiento principal expresado por las grandes marcas es en el núcleo poblacional de entre 15-40 años; y los menores de 15 años pueden estar alterados por otros factores más allá de lo que tienen en su mente sobre las marcas, es decir, para los adolescentes muchas veces es más importante vestir como viste el resto, que el propio gusto de cada uno basado en lo que piensa realmente de la marca, lo que restaría objetividad al estudio.

El cuestionario se ha distribuido digitalmente mediante el aplicativo gratuito de Google para realizar encuestas online: Google Formularios. De esta forma ha sido mucho más fácil la transmisión de las preguntas al usar un canal de fácil uso, acceso y envío. Dado que no había ninguna exigencia metodológica que cumplir, el Formulario se envió aleatoriamente a toda aquella persona mayor de 15 años (en caso de filtrarse alguna persona menor a esa edad quedaría reflejado en la pregunta "Edad", donde se quedaría sin opción a respuesta) sin distinción de sexo, antecedentes deportivos, lugar de residencia, etc....

El cuestionario es completamente anónimo y consta de muy pocas preguntas para facilitar y motivar su respuesta (10 preguntas). Sin embargo, lo interesante de este cuestionario es que de cada pregunta se saca información valiosa que analizar. Para poder sacar esos datos objetivos y concretos, realizaremos algunas preguntas que sirvan para entender, por ejemplo, el "Top of mind" que los consumidores tienen en su mente sobre las marcas. Recordemos que se trata de establecer el lugar que ocupan las marcas en nuestro cerebro, no a sus productos. También hay que tener claro que tener la primera posición en la mente de un consumidor no garantiza que este adquiera sus productos, ya que en la toma de decisión final 
repercuten otros factores externos: precio, distribución... Pero sin duda es un método eficaz para entender el posicionamiento que tienen las marcas en la mente. Los datos conseguidos posteriormente han sido analizados mediante Excel para poder tener las todas las estadísticas de los resultados. A continuación, se explica con mayor exactitud la estructura completa del cuestionario y el objetivo de cada pregunta formulada.

\section{Resultados.}

\section{NIKE, Inc.:}

Es la marca líder indiscutible en el mercado deportivo. Nace en 1964 en Oregón (EEUU). Vende zapatillas, textil, equipamiento, accesorios y servicios. Y dentro del portafolio de marcas incluidas a Nike encontramos: Jordán Brand, Hurley y Converse.

Target cliente: Podemos decir de antemano que Nike es una marca para todos. El sentido es que busca satisfacer las necesidades de todo el mundo, da igual la edad, sexo, país... Adaptan productos a las costumbres de muchas culturas (el cricket no se vende de igual forma en la India que en España, pero su target Core es completamente global). Cubren desde jóvenes hasta mayores (principalmente 18-40 años), solteros o casados, cualquier clase socioeconómica, deportistas o moda casual... Y prueba de este target son sus voluminosos números de ingresos, significativo de que todo el mundo está dispuesto a comprar Nike, o al menos eso es lo que desea la marca. Lo que sí que es cierto, es que su campaña actual de audiencia se basa en tres grupos de población principales:

1) El primero, y que todas las marcas están explotando y dimensionando al máximo actualmente, es el de las mujeres. Tal y como explica el CEO de la marca Mark Parker, y cuyas palabras quedan reflejadas en el artículo de Ashley Lutz (business insider, 2015), ya en 2015, en el primer trimestre, alcanzaron más ventas en material de entrenamiento y running para mujeres que en los hombres; y estima que para 2017 las ventas de Nike en mujeres sean 2 billones de US\$ más de lo que se vende actualmente (5.72 billones US\$ en 2015 y 4.97 billones US\$ en 2014). Actualmente, y como veremos en el apartado "Reason to believe", la marca ha iniciado a usar como imagen a grandes estrellas del mundo del deporte femenino como parte de su estrategia de implantación.

2) El segundo grupo principal al que está focalizando su atención actualmente Nike es el de jóvenes atletas. Niños que jueguen ya con los productos de Nike, garantiza que crecerán con ellos y seguirán consumiéndolos. La marca actúa patrocinando y utilizando la imagen de famosos deportistas que sirven de inspiración y referencia a esos jóvenes atletas que un día querrán ser como ellos.

3) El tercer grupo en el que la empresa basa su estrategia es el de los "runners". Tal y como explica su CEO, Parker, muchos de los esfuerzos en innovación de la marca van enfocados al mundo del running. En el último año fiscal, la marca ha ingresado 
4.853 millones de US\$ en concepto de Running; casi el 19\% del total de ingresos que obtiene la marca por sus secciones deportivas (Basketball el 14.4\%, Fútbol el 8.7\%, etc... Su imagen en miles de eventos del mundo del running es ya algo normal, y siempre buscan el acercamiento con el consumidor en ese sector

Definición de mercado: En cuanto a distribución geográfica, Nike basa su centro de comercialización en Estados Unidos, el lugar donde nació la marca. En el último año fiscal obtuvo unos ingresos de 13.740 millones de US\$ en Norte América (47.9\% del total de ingresos global), creciendo un $12 \%$ respecto al año 2014. Sin embargo, el mercado donde más creció en 2015 respecto al año anterior fue en China (+18\% y con un $11 \%$ de la cuota de ingresos total). Es Europa Occidental el segundo mercado con mayores ingresos para la marca (5.709 millones de US\$; casi un 20\% del total de ingresos) y que también ha crecido sustancialmente respecto al 2014 (+15\%). Además, es notable mencionar que desde 2013 han aumentado un 34\% los ingresos en Europa Occidental; lo que hace entender su gran apuesta por ese mercado. En cuanto a sus productos, la marca se basa en tres líneas generales: Zapatillas, textil y equipamiento deportivo, siendo el de las zapatillas su mercado estrella ocupando siempre el primer lugar. Basándonos en los ingresos, el mercado de las zapatillas ocupa un $63.8 \%$ de cuota; el textil un $30.1 \%$ y el de equipamiento deportivo un $5.7 \%$. Basándonos en las categorías deportivas principales en los que entra en mercado, el primer lugar lo ocupa el Sportswear (25.5\%), ofreciendo al consumidor ropa deportiva "fashion" bajo diseños muy bien estudiados; en segundo lugar aparecería el Running (18.8\% del total de ingresos de la marca), seguido de Baloncesto (14.4\%), Men's Traininng (9.8\%), Fútbol (8.7\%), Women's Training (4.9\%), Deportes de acción (2.8\%), y Golf (3\%).

Brand promise: La marca habla de que su misión se basa en "Dar inspiración e innovación a todos los atletas del mundo" ("To bring inspiration and innovation to every athlete in the world"). La marca basa su promesa en que el consumidor adquirirá un producto creado con técnicas innovadoras otorgándole una alta calidad, y bajo un gran control del diseño (incluido el de los materiales). Una de las técnicas de marketing que utilizó el Cofundador de la empresa, Bill Bowerman, para transmitir esos beneficios, y que hoy en día sigue utilizándose como eslogan en muchas campañas publicitarias de la marca fue: "Si tienes un cuerpo, eres un atleta" ("If you have a body, you are an athlete).Más allá del sentido de marketing de la frase, vemos como la promesa de Nike va encaminada a todo el mundo (tal y como he mencionado en el apartado de Target cliente), ya que todo el mundo que tenga un cuerpo puede ser atleta y usar sus productos innovadores.

Reason to believe: La manera que tiene Nike de mantener su posicionamiento global es a través de programas de implantación de marketing basados en anuncios, campañas con 
los consumidores, innovación del producto y calidad del producto. La empresa norteamericana fue pionera en la utilización de estrellas deportivas para promocionar su marca con el "fichaje" de Michael Jordán en 1984 bajo el contrato más alto jamás hecho anteriormente (500.000\$/temporada) y personalizando y explotando un producto denominado: Air Jordán (actualmente la marca Jordán es una línea de producto de éxito de la propia Nike). Hoy en día Nike tiene como embajadores de la marca a muchas de las estrellas deportivas del mundo: Cristiano Ronaldo, Kobe Bryant, Roger Federer, Lebrón James, Tiger Woods, Rory Mcllroy, Rafael Nadal, etc. Llevando el patrocinio de figuras mundiales del deporte al campo estratégico de actuación contra la competencia. La lucha entre Adidas y Nike se ve reflejada también en este campo: FC Barcelona (Nike) vs Real Madrid CF (Adidas); Rafael Nadal (Nike) vs Tomás Berdych (Adidas), Cristiano Ronaldo (Nike) vs Leo Messi (Adidas).

Hoy en día, la marca estadounidense está basando su patrocinio en busca de estrellas del deporte femenino que sean referencia mundial, en busca de ampliar y expandir sus productos a las mujeres. Prueba de ello son los contratos que tiene con dos de las mejores atletas del momento: Allyson Félix y Dafne Schippers. También el que tenía con María Sharapova dado su liderazgo en el tenis mundial, pero cuyo contrato fue rescindido debido a su positivo por sustancias prohibidas en marzo de 2016. Otras mujeres "estrella" que tienen contratos con la marca americana son: Hope Solo (fútbol), Serena Williams (Tenis) o Michelle Wie (Golf). Pero sin duda, una de las "Reason to believe” más destacadas y conocidas de la marca norteamericana es su eslogan "Just do it", utilizado por primera vez en 1988 y que hoy en día es emblema de la empresa y base de sus campañas publicitarias. Como había explicado anteriormente, la capacidad de una marca de que el consumidor sea capaz de reconocer y asociar un nombre a un posicionamiento es básico para la supervivencia de la misma. Sin duda, la marca ha alcanzado el éxito con este eslogan, logrando que el consumidor piense en Nike cuando oye o ve esa frase y sea capaz de asociarla a términos como: La victoria, la superación o la profesionalidad.

\section{PUMA:}

Es una de las empresas deportivas más importantes del mundo como hemos visto en el análisis de las marcas deportivas. Fue creada en 1948 de la mano del alemán Rudolf Dassler (hermano de Adolf, fundador de Adidas). En el pasado año fiscal la marca aumentó sus ingresos hasta los 3.89 billones de US\$. Dato muy relevante dado que en los ingresos de 2011 apenas llegaban a los 3 billones de US\$. En tres años han conseguido aumentar sus ventas en más del $20 \%$ y esto es en gran parte debido a su estrategia de reposicionamiento introducida en la marca en 2013 y que afectó a todos los procesos de Puma. La empresa alemana cuenta también en sus filas con la marca especializada en golf: Cobra Golf.

Target cliente: Al igual que ocurría con Nike, nos encontramos con una marca global que distribuye a más de 120 países y que, por lo tanto, no puede descartar ningún grupo 
de población al vender productos deportivos para todos. Sin embargo, sí que podemos concretar que su target Core cliente es una persona joven de entre 15-30 años, que no distingue entre sexos, religiones o aspectos socioeconómicos. La gente joven es mucho más social que otros grupos socio demográficos, y eso es lo que le gusta a Puma, gente con un lado salvaje y dinámico con los que asociar sus productos. Tal y como expliqué en el posicionamiento de Nike, las marcas deportivas más potentes saben que hoy en día se debe explotar el mercado femenino, y Puma ha sido de las pioneras en este segmento que ha establecido ya acuerdos y estrategias para potenciar sus productos al target mujer desde hace años. En 2016, la marca alemana ha introducido una colección completa y nueva para mujeres en zapatillas y textil, siendo el pilar de su nueva oferta de productos para mujeres. Asimismo, y con el fin de expandir su marca al sector femenino, cuenta desde 2015 con la artista internacional Rihanna como embajadora de la marca y directora creativa de muchos de sus productos (ej. Creeper).

Mercado: Puma distribuye a más de 120 países en el mundo, y su estrategia principal debe ser igual para todos. Al ser una marca nacida en Alemania, su mercado principal siempre era el europeo. En la región EMEA (Europa, Oriente Medio y África) tuvieron un 4.3\% más de ingresos respecto al año 2014 (1.258.2 millones de $€$ ) que representaron el $37.1 \%$ de las ventas totales (el año 2014 tuvieron el 40.6\%). En América, los ingresos aumentaron un $22.5 \%$ hasta los $1.310,8$ millones de $€$, lo que hace que represente el $38.7 \%$ de las ventas totales de la marca, superando por primera vez al mercado europeo (el año pasado obtuvo el $36 \%$ de las ventas totales). En cuanto a Asia/Pacífico, también han tenido un notable ascenso de las ventas con 818.4 millones de $€(+17.5 \%)$, lo que representa el $24.2 \%$ de las ventas totales de la marca, siendo China e India los países que mayor éxito han logrado.

Brand promise: Como he comentado anteriormente, Puma introduce una estrategia de reposicionamiento de la marca a nivel global en 2013 con el objetivo de modificar percepciones que tengan sus consumidores y así aumentar los ingresos, como así se ha demostrado en este último año fiscal. Bjørn Gulden, CEO de la marca, introduce la nueva misión de Puma que debe extrapolarse a cualquier proceso dentro de la marca: "To be the Fastest Sports Brand in the World" (Ser la marca deportiva más rápida del mundo). La empresa quiere expresar con esta promesa de marca todas sus acciones y decisiones. El objetivo de este reposicionamiento es demostrar que Puma vuelve de nuevo al deporte con una actitud distintiva: valiente, confiada, determinada y alegre. Sus productos son ligeros, cómodos y dinámicos; siendo esta vez mucho más innovadores y con diseños más claros. Todo ello bajo sus tres valores centrales: Ser sostenible a nivel social, económico y medioambiental. Su promesa es que producen los productos más innovadores para los atletas más rápidos del planeta. Y todo consumidor puede ser atleta y puede ser el más rápido. 
Reason to believe: La marca alemana refleja su promesa en el eslogan "Forever Faster", y que quiere acercar al público su idea de que sus productos están hechos para gente que quiere ser la más rápida usando tecnología innovadora. Un ejemplo tangible (y "Razón para creer" en su promesa) de su reposicionamiento que quiere demostrar ser el más rápido entre todas las marcas deportivas son sus productos, los cuales muchas veces son la imagen directa frente al consumidor. En este año Puma ha introducido un nuevo producto en el fútbol llamado evo SPEED, que ha conseguido crear las zapatillas de fútbol más ligeras del mercado (103 gramos). O su última innovación en running: IGNITE, que mejora la recuperación de energía y promocionado por el atleta más rápido de todos los tiempos: Usain Bolt. También, como parte de su imagen y fiel reflejo de este nuevo posicionamiento que están desarrollando, la marca ha implantado diversas tiendas completamente nuevas bajo el concepto Forever Fast. En ellas se optimiza la presentación de sus productos y sus tecnologías y fortalezas asociadas a la marca. Así mismo, lanzaron una renovada 'web shop' en 2015 que les permite expandirse en el mercado online y acceder a otros mercados distintos. La forma más generalizada de la marca para expandir su nuevo posicionamiento es mediante campañas publicitarias donde utilizan a las estrellas deportivas "más" rápidas, "más" fuertes, "más" goleadoras, "más" Y demuestran como entrenan con sus productos. Prueba de ello es la promoción de su producto estrella en el Running: Ignite mediante anuncios utilizando a Usain Bolt, su embajador principal.

\section{Conclusiones.}

- Nike es líder indiscutible en notoriedad, tenga la edad que tenga el sujeto. El grupo de edad de entre 26 y 35 años son los que mayor número de marcas deportivas diferentes ofrecen, lo que hace entender una mayor exposición a lo largo de su vida de impactos de muchas marcas diferentes. El grupo de edad de 51 años o más son los que menor número de marcas deportivas diferentes ofrecen (el boom de la publicidad no era tan grande ni agresivo como lo es actualmente). La muestra de este grupo de edad fue $\mathrm{N}=8$ sujetos, lo que obliga a ampliar estos resultados en futuros estudios. Además, tiene un nivel de atractivo alto, ya que, el 70\% de los encuestados consiguieron asociar correctamente los beneficios que estos piensan que tienen sus productos, con la promesa de marca que realmente quiere transmitir la propia Nike.

- Puma se consolida como tercera marca en notoriedad después de Nike y Adidas en todos los grupos de edad menos en los mayores de 51 años donde la más mencionada ha sido Reebok. Puma se afianza como tercera marca, desbancando a Asics en atletismo y a Reebok en fútbol. El atletismo hay mucha más variedad de marcas que atraen consumidores de ese deporte que en el fútbol. Puma tiene un nivel de atractivo muy bajo, ya que, el 8\% de los encuestados consiguieron asociar correctamente los beneficios que estos piensan que tienen sus productos, con la promesa de marca que realmente quiere 
transmitir la propia Puma. Puma tiene un nivel de atractivo muy bajo, ya que, el $8 \%$ de los encuestados consiguieron asociar correctamente los beneficios que estos piensan que tienen sus productos, con la promesa de marca que realmente quiere transmitir la propia Puma.

- Mucha gente se ha guiado por la notoriedad que tienen las marcas y queda reflejado en el alto número de sujetos que han mencionado a Adidas en ambas promesas de marca o como Nike consigue ser la marca más votada también en la promesa que quiere transmitir Puma.

- Nike y Adidas, pero sobre todo Puma deben comunicar mejor sus beneficios como marca para que su imagen como marca sea correctamente posicionada en la mente de los consumidores.

\section{Referencias Bibliográficas}

Consejo Superior de Deporte, CSD (2015). Memoria 2015: Licencias y clubes federados. Recuperado el 15 de abril de 2016 de: http://www.csd.gob.es/csd/estaticos/asoc-fed/LicenciasyClubes2015.pdf

Kearney, A.T. Inc. (2014). Winning in the Business of Sports. Recuperado el 13 de abril de 2016

Murphy, J. (1988). Branding. Marketing Intelligence \& Planning, Vol. 6 Iss 4 pp. $4-8$.

Ratten, V. and Ratten, H. (2011), International sport marketing: practical and future research Implications. Journal of Business \& Industrial Marketing, Vol. 26 No. 8, pp. 614-620.

Ratten, V. (2016). The dynamics of sport marketing. Marketing Intelligence \& Planning, Vol. 34 Iss 2 pp. $162-168$.

Ries, A. \& Trout, J. (2008). Positioning. The battle for your mind. New York: Warner Books - McGraw-Hill Inc.

Rom, J. \& Sabaté, J. (2007). Language publicities. Strategic i creative publicities. Girona, Universitat Oberta de Catalunya, p. 131.

Smith, A. (2008). Introduction to sport marketing. Sport Management Series. BH.

Trout, J. (1969). Positioning is a game people play in today's me-too marketplace. Industrial Marketing, n. 54, p. 51-55.

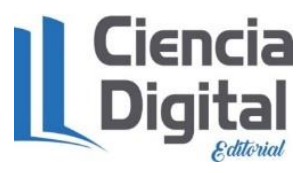




\section{PARA CITAR EL ARTÍCULO INDEXADO.}

Ortiz Gonzales, D., Torres Cedeño, M., \& Intriago Mairongo, H. (2019). El posicionamiento de una marca deportiva (Brand positioning). Visionario Digital, 3(3), 63-74. https://doi.org/10.33262/visionariodigital.v3i3.611

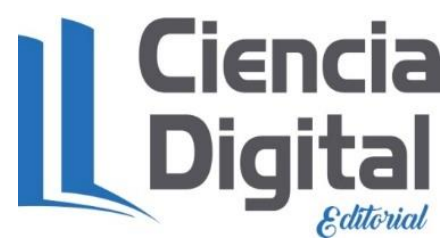

El artículo que se publica es de exclusiva responsabilidad de los autores y no necesariamente reflejan el pensamiento de la Revista Visionario Digital.

El artículo queda en propiedad de la revista y, por tanto, su publicación parcial y/o total en otro medio tiene que ser autorizado por el director de la Revista Visionario Digital.
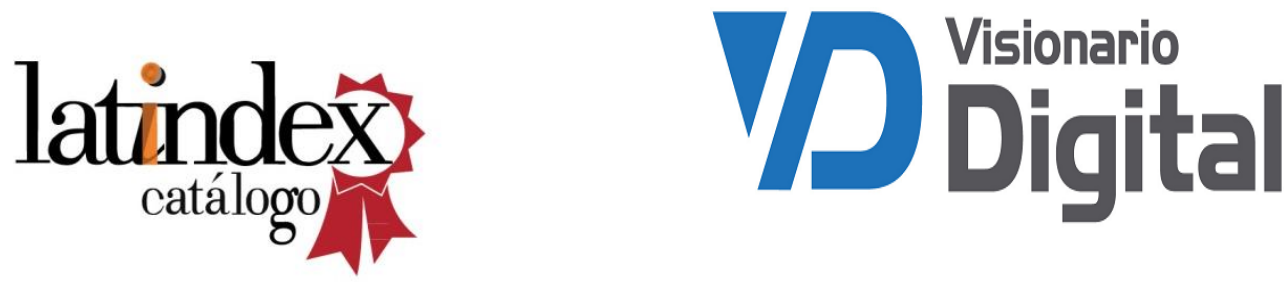